\title{
Decreased analgesic requirement in recipient of liver transplantation from monozygotic twin - A case report -
}

Received May 2, 2019

Revised 1st, June 24, 2019

2nd, July 14, 2019

Accepted September 5, 2019

\section{Corresponding author}

Gaab-Soo Kim, M.D., Ph.D.

Department of Anesthesiology and

Pain Medicine, Samsung Medical

Center, Sungkyunkwan University

School of Medicine, 81 Irwon-ro,

Gangnam-gu, Seoul 06351, Korea

Tel: 82-2-3410-0360

Fax: 82-2-3410-0361

E-mail: gskim@skku.edu

ORCID

https://orcid.org/0000-0002-1563-4482

\author{
Ah Ran Oh, Justin Sangwook Ko, and Gaab-Soo Kim \\ Department of Anesthesiology and Pain Medicine, Samsung Medical Center, \\ Sungkyunkwan University School of Medicine, Seoul, Korea
}

Background: There have been many reports about decreased analgesic requirements in liver transplant recipients compared with patients undergoing other abdominal surgery.

Case: Herein we describe a case in which a 42-year-old man underwent living donor liver transplantation from his monozygotic twin. Because innate pain thresholds may be similar in monozygotic twins, we could effectively investigate postoperative pain in the donor and the recipient. Concordant with previous reports, the recipient used less analgesic than the donor in the present study.

Conclusions: Physicians caring for patients who have received liver transplantation should consider their comparatively low requirement for analgesic, to prevent delayed recovery due to excessive use of analgesic.

Keywords: Analgesics; Liver transplantation; Monozygotic twin.
It has been reported that liver transplantation (LT) recipients require less pain control postoperatively than patients undergoing other abdominal surgery [1-3]. This is important because inappropriate use of analgesic after LT may result in excessive sedation and delayed weaning from mechanical ventilation [4]. Although elevated endogenous opioids during end-stage liver disease was thought to be the cause of decreased analgesic requirement in LT recipients, the precise mechanism has not been clarified [5]. Recently, we performed living donor LT between monozygotic twins. Because pain thresholds might be similar in monozygotic twins due to their near complete genetic similarity, we could compare the analgesic requirements of the LT recipient with those of the donor with reduced influence of differences in innate pain thresholds.

\section{CASE REPORT}

A 42-year-old man (height $178 \mathrm{~cm}$, weight $73 \mathrm{~kg}$ ) was admitted for LT due to recurrent hepatitis B virus-related hepatocellular carcinoma. He had undergone right posterior sectionectomy 7 months prior and trans-arterial chemoembolization twice. Despite these treatments he was diagnosed with recurrent hepatocellular carcinoma; therefore, he was scheduled for LT. His model for end-stage liver disease score was 14, and preoperative laboratory results were almost normal with the exception of a low platelet count $\left(105 \times 10^{3} / \mu \mathrm{l}\right)$. On the day of surgery, pulse oximetry, five lead electrocardiography, and non-invasive blood pressure monitoring were applied. General anesthesia was induced via $350 \mathrm{mg}$ thiopental sodium and $40 \mathrm{mg}$ atracurium, followed by endotracheal intubation. Hemodynamic monitoring included direct arterial pressures from the right radial artery and right femoral artery, central venous pressures from the right femoral vein and right inter-

This is an Open Access article distributed under the terms of the Creative Commons Attribution Non-Commercial License (http://creativecommons.org/licenses/by-nc/4.0) which permits unrestricted non-commercial use, distribution, and reproduction in any medium, provided the original work is properly cited.

Copyright (c) the Korean Society of Anesthesiologists, 2020 
nal jugular vein, and cardiac output and pulmonary artery pressure from a Swan-Ganz catheter. Anesthesia was maintained with remifentanil and isoflurane. Surgery was started with a bilateral subcostal incision extending to the cephalic side, and he underwent the operation with stable vital signs. The total anesthesia time was $606 \mathrm{~min}$. The infused crystalloid solutions were 6,300 ml of Plasma solution A (CJ HealthCare Corp., Korea) and $240 \mathrm{ml}$ of $5 \%$ dextrose solution. The infused colloid solutions were $1,020 \mathrm{ml}$ of $5 \%$ albumin (Green Cross Corp., Korea) and 1,000 ml of Hextend (CJ HealthCare Corp.). Auto-transfusion $(200 \mathrm{ml})$ was performed via intraoperative blood salvage, and urine output was $880 \mathrm{ml}$. After the operation he was transferred to the intensive care unit and the endotracheal tube was extubated on the afternoon of the following day.

The donor (height $177 \mathrm{~cm}$, weight $70 \mathrm{~kg}$ ) was the recipient's monozygotic twin, and he was healthy without any underlying disease. After applying standard monitoring he was administered $400 \mu \mathrm{g}$ morphine sulfate (BC World Pharm Co., Ltd., Korea) via intrathecal injection at lower lumbar level for
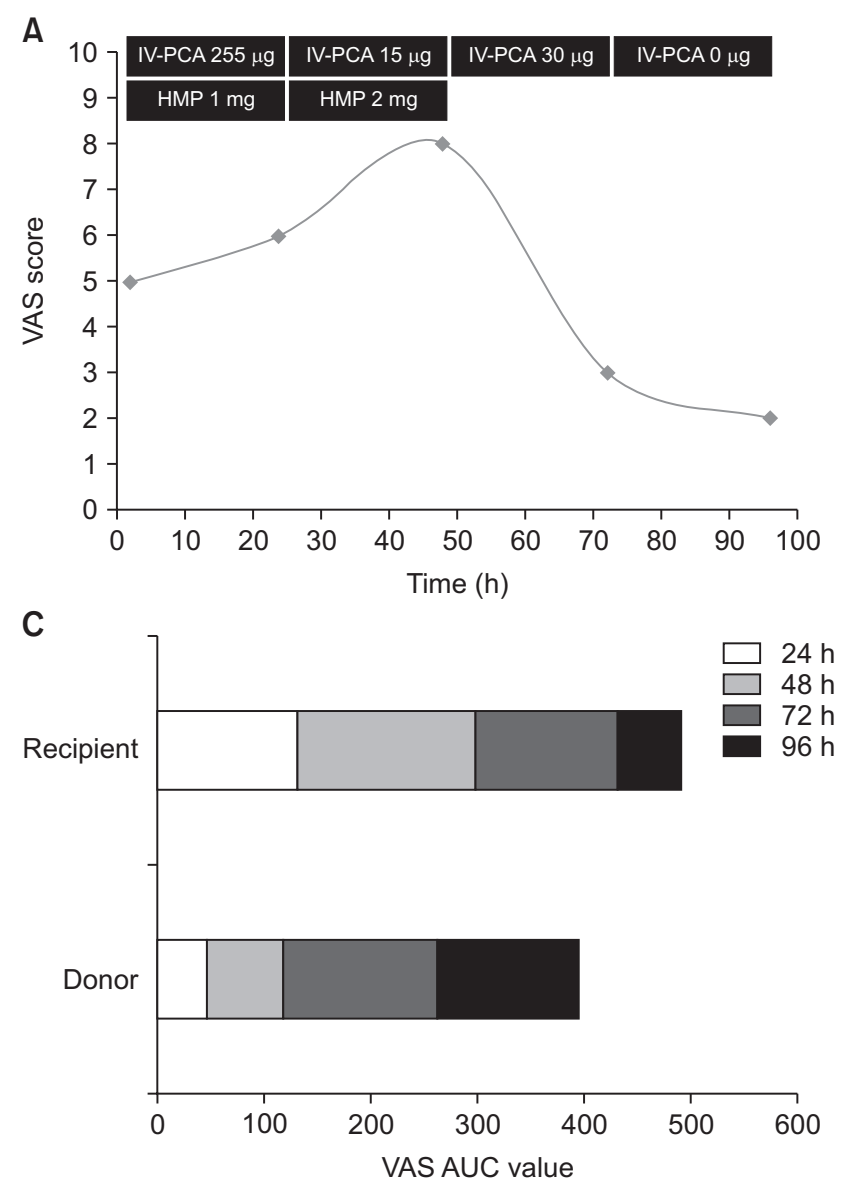

postoperative pain control. General anesthesia was induced via $350 \mathrm{mg}$ thiopental sodium and $8 \mathrm{mg}$ vecuronium followed by supraglottic airway insertion (Protector ${ }^{\mathrm{TM}}$ airway with cuff pilot technology, size 4, Teleflex Medical Europe, Ltd., Ireland). Anesthesia was maintained with remifentanil and isoflurane. The operation was started with laparoscopic surgery but it was changed to open bilateral subcostal incision due to portal vein variation. His vital signs remained stable during the surgery and he was administered pethidine $25 \mathrm{mg}$ when the surgeon closed the abdominal fascia. Total anesthesia time was $360 \mathrm{~min}$. Plasma solution A (1,950 ml) and $500 \mathrm{ml}$ colloid solution Volulyte (R) 6\% (Fresenius Kabi Pharmaceutical, Germany) were infused during surgery. Total urine output was $235 \mathrm{ml}$. After the operation he was transferred to the post-anesthesia care unit and the supraglottic airway was removed.

Intravenous patient-controlled analgesia (IV-PCA) was used for postoperative pain control, and it was started when each patient requested pain control. Each patient received the same fentanyl (Hana Pharmaceutical Cp., Ltd., Korea)-

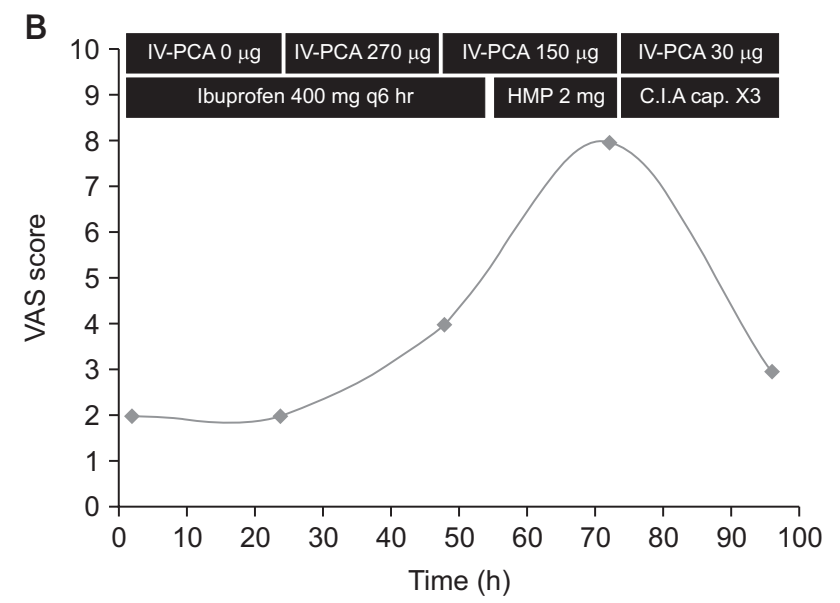

Fig. 1. (A) The liver transplant recipient's visual analogue scale scores and analgesic administered over a 96-h period postoperatively. (B) The liver transplant donor's visual analogue scale scores and analgesic administered over a 96-h period postoperatively. (C) Visual analogue scale area under the curve values in the donor and the recipient over a 96-h period postoperatively. VAS: visual analogue scale, IV-PCA: intravenous patient-controlled analgesia, HMP: hydromorphone, C.I.A Cap.: codeineibuprofen-acetaminophen capsule, AUC: area under the curve. 
based IV-PCA with bolus $15 \mu \mathrm{g} / \mathrm{ml}$ and a lockout interval of 15 min without basal infusion. Hydromorphone (Hana Pharmaceutical Cp., Ltd.) was injected for supplementary analgesia when patients suffered from pain that was reportedly not controlled by IV-PCA. Hydromorphone was only given when the visual analogue scale (VAS) score was 4 or more. In the donor, $400 \mathrm{mg}$ of ibuprofen (Huons Co., Ltd., Korea) was injected regularly on postoperative days (PODs) 1,2 , and 3 and capsules composed of codeine phosphate $10 \mathrm{mg}$, ibuprofen $200 \mathrm{mg}$, and acetaminophen $250 \mathrm{mg}$ (C.I.A Capsules, Myungmoon Pharmaceutical Cp., Ltd., Korea) were given on POD 4 when he required pain control. For postoperative pain evaluation the patients were visited by an anesthesiologist once a day for a period of 4 days. Data including IV-PCA use, the use of other analgesics, and VAS scores were collected up to and including POD 4 (Fig. 1A, B). The VAS area under the curve (AUC) value is shown in Fig. 1C. Total opioid analgesic use expressed in morphine equivalent dose is shown in Fig. 2. All information described in this case report was collected with the informed consent of the donor and the recipient.

\section{DISCUSSION}

LT is very extensive abdominal surgery in terms of duration and surgical stress for the patient. A large bilateral subcostal incision extending to the xiphoid process, and a surgical retractor that pulls the incision contribute to postoperative pain. Post-LT pain is not as severe as might be expected in recipients however, and decreased analgesic requirement

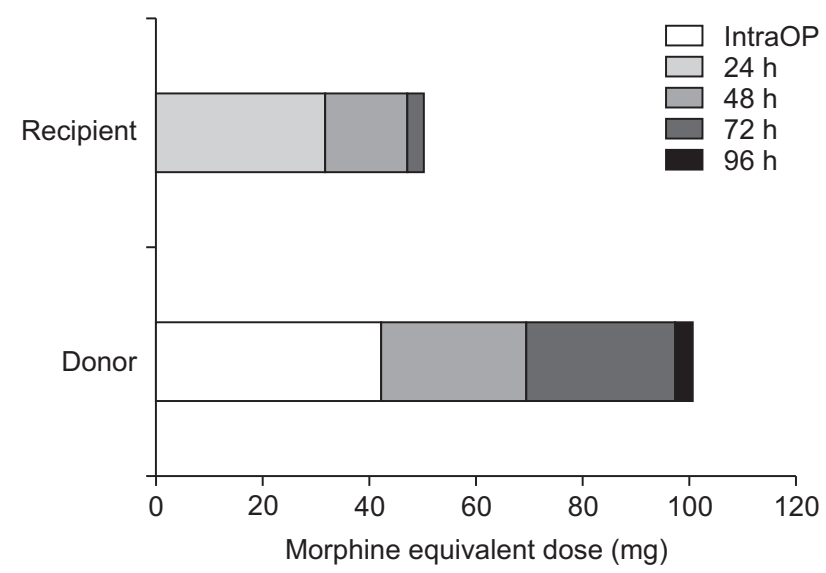

Fig. 2. Total perioperative opioid consumption in the donor and the recipient, expressed as intravenous morphine equivalent dose. IntraOP: intraoperative. has been reported in recipients. In the present case we could compare postoperative pain and analgesic requirements in a living LT donor and the LT recipient with reduced influence of individual differences in innate pain thresholds due to their near complete genetic similarity.

Postoperative pain is severe immediately after surgery. The VAS score of the recipient in the present case was high on PODs 1 and 2, then gradually decreased (Fig. 1A). Conversely the VAS score of the donor increased after POD 2 (Fig. 1B). This unusual pattern in the donor's VAS scores is probably due to the intrathecal morphine, the analgesic effects of which lasted until POD 2 [6]. Although the actual VAS AUC value was lower in the donor than in the recipient (Fig. 1C), it is reasonable to assume that the VAS AUC value would have been larger in the donor than in the recipient were it not for the effects of intrathecal morphine [6]. The amounts of opioids administered during intraoperative and postoperative periods were converted to intravenous morphine equivalent doses (intravenous morphine:intrathecal morphine:pethi dine:fentanyl:hydromorphone = 1.00:0.10:10.00:0.01:0.15) (Fig. 2). Even if the non-opioid analgesic is excluded, the total amount of analgesic use was less in the recipient than in the donor.

We can suggest several reasons for the comparatively lower analgesic requirement in an LT recipient. One is that pharmacokinetic changes in patients with end-stage liver disease may affect the metabolism of drugs. Decreased hepatic blood flow, drug-metabolizing enzyme activity, and drug-bound plasma protein could be contributing factors $[7,8]$. Notably however, hepatic blood flow of the graft and plasma albumin were normal postoperatively in the recipient. Moreover, there was no evidence of non-function or dysfunction of newly transplanted liver. Thus, pharmacokinetic change could not explain the comparatively lower postoperative analgesic requirements in the recipient. Another possible reason for the lower postoperative analgesic requirements is that interaction between immunosuppressive agents and opioid receptors may alter the efficacy of analgesics. In particular, the effects of cyclosporine A on analgesic requirements have been extensively studied. During the inflammatory phase, cytokines may evoke opioid-induced anti-nociception in peripheral opioid receptors due to the release of endogenous opioids via interleukin 1 beta and corticotropin-releasing factor [9]. However, this effect could be reversed by cyclosporine 
A due to interactions involving opioid receptors [10]. Furthermore, Wolkowitz et al. [11] suggested that repeated administration of prednisolone leads to reduced beta-endorphin in human cerebrospinal fluid. Therefore immunosuppressive agents may not have contributed to the LT recipient requiring comparatively less analgesic than the donor in the present case.

A third possibility relates to a report by Donovan et al. [5], who suggested that increased plasma levels of endogenous neuropeptides could contribute to modulation of pain in chronic liver disease. Metenkephalin and beta-endorphin have been shown to exert analgesic effects via direct binding to opioid receptors. They measured plasma metenkephalin and beta-endorphin levels for 3 days after surgery in $13 \mathrm{LT}$ recipients and 10 control patients. Metenkephalin levels were elevated in all LT recipients when compared with control patients, but beta-endorphin levels were not. That result suggests that increased metenkephalin levels in chronic liver disease may contribute to decreased analgesic requirements in LT recipients. Therefore, it is reasonable to surmise that increased endogenous opioids in end-stage liver disease may contribute to a higher pain threshold in LT recipients than in LT donors.

Analgesic use in LT recipients is an important consideration with regard to rapid recovery. Jullien et al. [12] asserted that spontaneous ventilation may be beneficial in hemodynamically stable transplant patients, promoting hepatic venous drainage and graft circulation. Postoperative positive ventilation induced by sedation due to excessive use of analgesic increases right ventricular workload, and backward flow into the inferior vena cava and hepatic veins resulting in venous congestion and poor graft circulation. Therefore, for rapid recovery of LT recipients, optimal use of low-dose analgesic to avoid over-sedation and facilitating early extubation should be a primary aim [13].

Although in the present case the LT recipient needed less analgesic than the donor after the surgery, there is one major limitation. It was not possible to accurately compare the VAS scores or total analgesic consumption of the two patients because of the different analgesic and pain control techniques used in each patient. However, the observations in the two patients are generally concordant with previous reports that LT recipients may tend to use less analgesic than LT donors during postoperative days.
In conclusion, in the present case involving an LT donor and an LT recipient who were almost genetically identical, the recipient required less analgesic than the donor for postoperative pain relief. Moreover, fine adjustment of the analgesic regimen is needed in both the LT recipient and the LT donor, based on the analgesic technique used.

\section{CONFLICTS OF INTEREST}

No potential conflict of interest relevant to this article was reported.

\section{ORCID}

Ah Ran Oh, https://orcid.org/0000-0002-8076-5104

Justin Sangwook Ko, https://orcid.org/0000-0003-3155-0550

\section{REFERENCES}

1. Moretti EW, Robertson KM, Tuttle-Newhall JE, Clavien PA, Gan TJ. Orthotopic liver transplant patients require less postoperative morphine than do patients undergoing hepatic resection. J Clin Anesth 2002; 14: 416-20.

2. Chen JP, Jawan B, Chen CL, Wang CH, Cheng KW, Wang CC, et al. Comparison of postoperative morphine requirements in healthy living liver donors, patients with hepatocellular carcinoma undergoing partial hepatectomy, and liver transplant recipients. Transplant Proc 2010; 42: 701-2.

3. Eisenach JC, Plevak DJ, Van Dyke RA, Southorn PA, Danielson DR, Krom RA, et al. Comparison of analgesic requirements after liver transplantation and cholecystectomy. Mayo Clin Proc 1989; 64: 356-9.

4. Pai SL, Aniskevich S, Rodrigues ES, Shine TS. Analgesic considerations for liver transplantation patients. Curr Clin Pharmacol 2015; 10: 54-65.

5. Donovan KL, Janicki PK, Striepe VI, Stoica C, Franks WT, Pinson CW. Decreased patient analgesic requirements after liver transplantation and associated neuropeptide levels. Transplantation 1997; 63: 1423-9.

6. Ko JS, Choi SJ, Gwak MS, Kim GS, Ahn HJ, Kim JA, et al. Intrathecal morphine combined with intravenous patient-controlled analgesia is an effective and safe method for immediate postoperative pain control in live liver donors. Liver Transpl 2009; 15: $381-9$.

7. Haberer JP, Schoeffler P, Couderc E, Duvaldestin P. Fentanyl pharmacokinetics in anaesthetized patients with cirrhosis. Br J 
Anaesth 1982; 54: 1267-70.

8. Wilkinson GR, Shand DG. Commentary: a physiological approach to hepatic drug clearance. Clin Pharmacol Ther 1975; 18: 377-90.

9. Schäfer M, Carter L, Stein C. Interleukin 1 beta and corticotropin-releasing factor inhibit pain by releasing opioids from immune cells in inflamed tissue. Proc Natl Acad Sci U S A 1994; 91: 4219-23.

10. Suzuki T, Yoshiike M, Funada M, Mizoguchi H, Kamei J, Misawa M. Effect of cyclosporine A on the morphine-induced place preference. Neurosci Lett 1993; 160: 159-62.
11. Wolkowitz OM, Rubinow D, Doran AR, Breier A, Berrettini WH, Kling MA, et al. Prednisone effects on neurochemistry and behavior. Preliminary findings. Arch Gen Psychiatry 1990; 47: 9638.

12. Jullien T, Valtier B, Hongnat JM, Dubourg O, Bourdarias JP, Jardin F. Incidence of tricuspid regurgitation and vena caval backward flow in mechanically ventilated patients. A color Doppler and contrast echocardiographic study. Chest 1995; 107: 488-93.

13. Mandell MS, Lezotte D, Kam I, Zamudio S. Reduced use of intensive care after liver transplantation: influence of early extubation. Liver Transpl 2002; 8: 676-81. 